\title{
Forced vibration analysis of laminated composite plates under the action of a moving vehicle
}

\author{
H.A. Mobaraki, R.-A. Jafari-Talookolaei \\ Babol Noshirvani University of Technology, Shariati Ave., 47148-71167 Babol, Mazandaran, Iran \\ Hossein.mobaraki1995@gmail.com, bttps://orcid.org/0000-0002-7713-1215 \\ ra.jafarinit.ac.ir,http://orcid.org/0000-0003-4357-2597
}

\section{P.S. Valvo}

University of Pisa, Largo Lucio Lazzarino, I-56122 Pisa, Italy

p.valvo@ing.unipi.it, bttp://orcid.org/0000-0001-6439-1926

\section{R. Haghani}

Chalmers University of Technology, Sven Hultins gata 6, SE-41296 Gothenburg, Sweden reza.haghani@chalmers.se, bttp://orcid.org/0000-0002-0547-7700

\begin{abstract}
This paper provides a finite element analysis of laminated composite plates under the action of a moving vehicle. The vehicle is modeled as a rigid body with four suspension systems, each consisting of a springdashpot. Overall, the vehicle possesses three degrees of freedom: vertical, rolling, and pitching motions. The equations of motion of the plate are deduced based on first-order shear deformation theory. Using the EulerLagrange equations, the system of coupled equations of motion is extracted and solved by using the Newmark time discretization scheme. The algorithm is validated through the comparison of both the free and forced vibration results provided by the present model and exact or numerical results reported in the literature. The effects are investigated of several system parameters on the dynamic response.
\end{abstract}

KEYWORDS. Forced Vibration; Laminated Composites; Moving Vehicle.

\section{OPEN ACCESS}

Citation: Mobaraki, H.A., Jafari-Talookolaei, R.-A., Valvo, P.S., Haghani Dogaheh, R., Forced vibration analysis of laminated composite plates under the action of a moving vehicle, Frattura ed Integrità Strutturale, 59 (2022) 198-211.

Received: 21.08 .2021

Accepted: 13.10 .2021

Published: 01.01.2022

Copyright: (C) 2022 This is an open access article under the terms of the CC-BY 4.0, which permits unrestricted use, distribution, and reproduction in any medium, provided the original author and source are credited.

\section{INTRODUCTION}

I $\mathrm{n}$ recent years, the dynamic analysis of engineering structures, such as bridges, roads, and rails, under the action of moving loads has gained great attention. Such structures are often subjected to high stresses and experience severe vibrations. Bridges as main substructures can be modeled as plates traversed by three major types of loading: moving loads, moving masses, and moving oscillators. Thus, researchers have studied their behavior under the action of these loadings. 
Some researchers have focused their studies on analyzing the effects of moving loads on isotropic beams and plates [1-4], while others have focused on the case of moving masses [5-8]. Besides, some works are related to moving oscillators [9-11]. Ghafoori et al. [12] provided a semi-analytical method to obtain the dynamic response of a plate subjected to a moving oscillator. Also, Wu et al. [13] aimed at introducing a technique to replace each 3-DOF system consisting of spring-mass by a set of equivalent masses. Lin and Trethewey [14] investigated the response of elastic beams subjected to an arbitrary springmass damper system and obtained the governing equations of motion based on the finite element method (FEM).

Recently, laminated composites, due to their lightweight and high strength, as well as the material adaptability, have gained in popularity for the construction of civil structures such as bridges. This has brought a new field of interest in studying the response of either laminated composite plates or beams traversed by moving oscillators or loads. Malekzadeh et al. [15] studied the dynamic response of cross-ply thick laminated plate under the action of moving load based on three-dimensional elasticity. They applied layerwise theory to discretize the equations of motion. Mohebpour et al. [16] investigated the response of laminated composite plate subjected to moving oscillator using the FEM based on first-order shear deformation theory (FSDT). In 2004, Lee et al. [17] analyzed a multi-span continuous composite plate under multi-moving loads based on third-order shear deformation theory (TSDT). Ghafoori and Asghari [18] presented an analysis of angle-ply laminated composite plates traversed by moving masses and forces. They applied the FEM to obtain equations of motion and solved them by using the Newmark method. Mohebpour et al. [19] developed an algorithm based on the FEM to study the response of laminated composite beams subjected to moving oscillators. They used FSDT to obtain the equations of the beam.

Kim [20] studied the dynamic stability behavior of damped laminated beams subjected to uniformly distributed forces based on a finite element formulation consistent with Vlasov's beam theory. Also, the effect of fiber orientation, boundary conditions, and external and internal damping was studied. It should be mentioned that the dynamic response of an intact plate can be used for damage detection in a defected plate [21-23].

In this paper, the problem of a laminated composite plate subjected to a moving vehicle is investigated. Thus, the effects of various parameters, such as vehicle mass, plate damping ratio, etc., are also investigated. The governing equations of the plate are obtained based on FSDT and the vehicle is considered as a rigid body having 3 degrees of freedom: vertical, rolling, and pitching motions. This modelling approach is the major novelty of the present paper. Lastly, the Newmark time integration procedure is used to find the response of the system in time.

\section{Mathematical Modeling}

onsider a laminated composite plate under the action of a moving vehicle with constant velocity $V$ along the $x$-axis as shown in Fig. 1. The plate has length $a$, width $b$ and thickness $b$ with the coordinate frame placed at the midplane. The displacement field based on FSDT is as follows:

$$
\begin{aligned}
& u(x, y, z, t)=u_{0}(x, y, t)+z \psi_{x}(x, y, t) \\
& v(x, y, z, t)=v_{0}(x, y, t)+z \psi_{y}(x, y, t) \\
& w(x, y, z, t)=w_{0}(x, y, t)
\end{aligned}
$$

where $u, v$ and $w$ are the displacements of a point of the laminate $(\mathrm{x}, \mathrm{y}, \mathrm{z})$ in the three coordinate directions. Also, $u_{0}$, $v_{0}$ and $w_{0}$ refer to displacements of a point on the mid-plane $(z=0)$ and $\psi_{x}, \psi_{y}$ refer to rotations about the $x$-and $y$ axis, respectively. Using Eqn.1, the non-zero strain components are derived as follow:

$$
\begin{aligned}
& \varepsilon_{x}=u_{, x}=u_{0, x}+z \psi_{x, x}=\varepsilon_{x}^{0}+z k_{x} \\
& \varepsilon_{y}=v_{, y}=v_{0, y}+z \psi_{y, y}=\varepsilon_{y}^{0}+z k_{y} \\
& \gamma_{x y}=u_{, y}+v_{, x}=u_{0, y}+v_{0, x}+z \psi_{x, y}+z \psi_{y, x}=\gamma_{x y}^{0}+z k_{x y}
\end{aligned}
$$




$$
\begin{aligned}
& \gamma_{x z}=\frac{\partial u}{\partial z}+\frac{\partial w}{\partial x}=\psi_{x}+\frac{\partial w_{0}}{\partial x} \\
& \gamma_{y z}=\frac{\partial v}{\partial z}+\frac{\partial w}{\partial y}=\psi_{y}+\frac{\partial w_{0}}{\partial y}
\end{aligned}
$$

where $\varepsilon_{x}, \varepsilon_{y}$, and $\gamma_{x y}$ denote the normal and shear strains of an arbitrary point, respectively. Furthermore, $\varepsilon_{x}^{0}, \varepsilon_{y}^{0}$, and $\gamma_{x y}^{0}$ are the mid-plane strains, and $k_{x}, k_{y}$ and $k_{x y}$ are the bending curvatures.

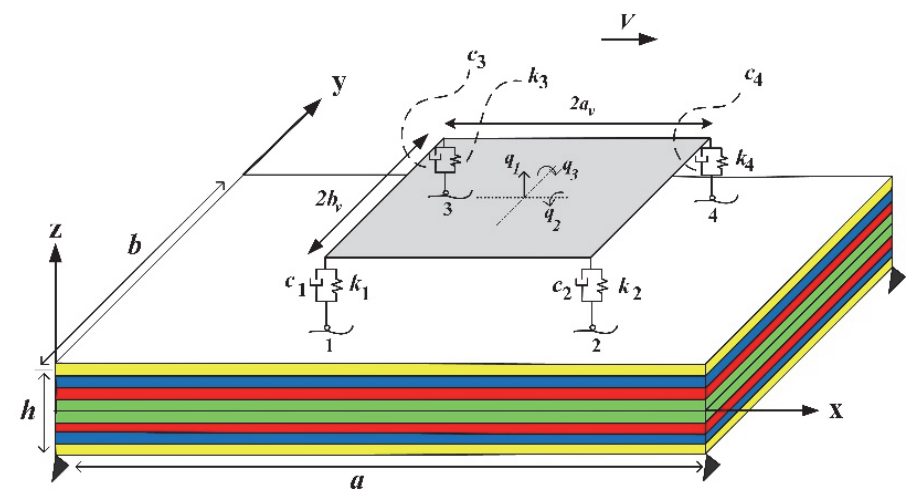

Figure 1: Laminated composite plate under the action of a moving vehicle

According to the transformed constitutive relations for a $2 \mathrm{D}$ orthotropic lamina, the stress-strain relations for the $k^{\text {th }}$ lamina can be written as [24]:

$$
\left\{\begin{array}{l}
\sigma_{x} \\
\sigma_{y y} \\
\tau_{x y}
\end{array}\right\}^{(k)}=\left[\begin{array}{lll}
\bar{Q}_{11} & \bar{Q}_{12} & \bar{Q}_{16} \\
\bar{Q}_{12} & \bar{Q}_{22} & \bar{Q}_{26} \\
\bar{Q}_{16} & \bar{Q}_{26} & \bar{Q}_{66}
\end{array}\right]^{(k)}\left\{\begin{array}{l}
\varepsilon_{x} \\
\varepsilon_{y} \\
\gamma_{x y}
\end{array}\right\}^{(k)},\left\{\begin{array}{l}
\tau_{y z} \\
\tau_{x z}
\end{array}\right\}^{(k)}=\left[\begin{array}{ll}
\bar{Q}_{44} & \bar{Q}_{45} \\
\bar{Q}_{45} & \bar{Q}_{55}
\end{array}\right]^{(k)}\left\{\begin{array}{l}
\gamma_{y z} \\
\gamma_{x z}
\end{array}\right\}^{(k)}
$$

where $\bar{Q}_{i j}$ is the reduced stiffness.

The strain energy of the laminated composite plate is:

$$
U_{P}=\frac{1}{2} \int_{0}^{a} \int_{0}^{b} \int_{-\frac{b}{2}}^{\frac{b}{2}}{ }^{b}\left(\sigma_{x}{ }^{(k)} \varepsilon_{x}{ }^{(k)}+\sigma_{y}{ }^{(k)} \varepsilon_{y}{ }^{(k)}+\tau_{x y}{ }^{(k)} \gamma_{x y}{ }^{(k)}+\tau_{y z}{ }^{(k)} \gamma_{y z}{ }^{(k)}+\tau_{x z}{ }^{(k)} \gamma_{x z}{ }^{(k)}\right) d z d y d x
$$

Substituting Eqn.3 into Eqn.4 and then integrating over the thickness leads to:

$$
\begin{aligned}
& \mathrm{U}_{\mathrm{P}}=\frac{1}{2} \int_{0}^{a} \int_{0}^{b}\left[A_{11} u_{0, x}^{2}+A_{22} v_{0, y}^{2}+A_{66}\left(u_{0, y}^{2}+v_{0, x}^{2}+2 u_{0, y} v_{0, x}\right)+D_{11} \psi_{x, x}^{2}+D_{22} \psi_{y, y}^{2}\right. \\
& +D_{66}\left(\psi_{x, y}^{2}+\psi_{y, x}^{2}+2 \psi_{x, y} \psi_{y, x}\right)+A_{44}\left(\psi_{y}^{2}+w_{0, y}^{2}+2 \psi_{y} w_{0, y}\right)+2 B_{16} \psi_{x, x}\left(u_{0, y}+v_{0, x}\right) \\
& +A_{55}\left(\psi_{x}^{2}+w_{0, x}^{2}+2 \psi_{x} w_{0, x}\right)+2 A_{12} u_{0, x} v_{0, y}+2 A_{16}\left(u_{0, y}+v_{0, x}\right) u_{0, x} \\
& +2 B_{11} \psi_{x, x} u_{0, x}+2 B_{12} \psi_{y, y} u_{0, x}+2 B_{16}\left(\psi_{x, y}+\psi_{y, x}\right) u_{0, x}+2 B_{26} \psi_{y, y}\left(u_{0, y}+v_{0, x}\right) \\
& +2 A_{26}\left(u_{0, y}+v_{0, x}\right) v_{0, y}+2 B_{12} \psi_{x, x} v_{0, y}+2 B_{22} \psi_{y, y} v_{0, y}+2 B_{26}\left(\psi_{x, y}+\psi_{y, x}\right) v_{0, y}
\end{aligned}
$$




$$
\begin{aligned}
& +2 B_{66}\left(\psi_{x, y}+\psi_{y, x}\right)\left(u_{0, y}+v_{0, x}\right)+2 D_{12} \psi_{y, y} \psi_{x, x}+2 D_{16}\left(\psi_{x, y}+\psi_{y, x}\right) \psi_{x, x} \\
& \left.+2 D_{26}\left(\psi_{x, y}+\psi_{y, x}\right) \psi_{y, y}+2 A_{45}\left(\psi_{x}+w_{0, x}\right)\left(\psi_{y}+w_{0, y}\right)\right] \mathrm{dy} \mathrm{dx}
\end{aligned}
$$

Furthermore, the kinetic energy of the laminated composite plate is:

$$
T_{P}=\frac{1}{2} \int_{0}^{a} \int_{0}^{b}\left[I_{0}\left(u_{, t}^{2}+v_{, t}^{2}+w_{, t}^{2}\right)+2 I_{1}\left(u_{, t} \psi_{x, t}+v_{,} \psi_{y, t}\right)+I_{2}\left(\psi_{x, t}^{2}+\psi_{y, t}^{2}\right) d y d x\right.
$$

where $\left(I_{0}, I_{1}, I_{2}\right)$ are mass moments of inertia, defined as follows:

$$
\left(I_{0}, I_{1}, I_{2}\right)=\int_{-b / 2}^{b / 2} \rho(z)\left(1, z, z^{2}\right) d z
$$

As mentioned earlier, a moving vehicle is modeled with 3-DOF as shown in Fig. 1. The potential energy of the vehicle is:

$$
\begin{aligned}
U_{V} & =\frac{1}{2} k_{1}\left(q_{1}-w_{1}-b_{V} q_{2}+a_{V} q_{3}\right)^{2}+\frac{1}{2} k_{2}\left(q_{1}-w_{2}-b_{V} q_{2}-a_{V} q_{3}\right)^{2} \\
& +\frac{1}{2} k_{3}\left(q_{1}-w_{3}+b_{V} q_{2}+a_{V} q_{3}\right)^{2}+\frac{1}{2} k_{4}\left(q_{1}-w_{4}+b_{V} q_{2}-a_{V} q_{3}\right)^{2}+\frac{m g}{4}\left(w_{1}+w_{2}+w_{3}+w_{4}\right)
\end{aligned}
$$

where

$$
w_{i}=w_{0}\left(x_{i}, y_{i}\right) \quad(i=1,2,3,4)
$$

Also, the damped energy of dashpots can be written as:

$$
\begin{aligned}
W_{d v} & =\frac{1}{2} c_{1}\left(\dot{q}_{1}-\dot{w}_{1}-b_{V} \dot{q}_{2}+a_{V} \dot{q}_{3}\right)^{2}+\frac{1}{2} c_{2}\left(\dot{q}_{1}-\dot{w}_{2}-b_{V} \dot{q}_{2}-a_{V} \dot{q}_{3}\right)^{2} \\
& +\frac{1}{2} c_{3}\left(\dot{q}_{1}-\dot{w}_{3}+b_{V} \dot{q}_{2}+a_{V} \dot{q}_{3}\right)^{2}+\frac{1}{2} c_{4}\left(\dot{q}_{1}-\dot{w}_{4}+b_{V} \dot{q}_{2}-a_{V} \dot{q}_{3}\right)^{2}
\end{aligned}
$$

In the above equations, $k_{i}$ and $c_{i}(i=1,2,3,4)$ refer to the suspension system stiffness and damping parameters, respectively. Moreover, the kinetic energy of the vehicle is:

$$
T_{V}=\frac{1}{2} m \dot{q}_{1}^{2}+\frac{1}{2} I_{x} \dot{q}_{2}^{2}+\frac{1}{2} I_{y} \dot{q}_{3}^{2}
$$

where $m$ is the vehicle total mass and $I_{x}$ and $I_{y}$ are its mass moments of inertia about the $x$-and $y$-axes respectively.

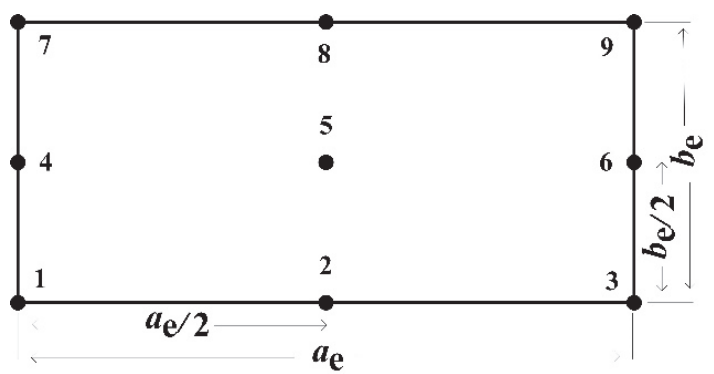

Figure 2: Rectangular higher-order element 


\section{FINITE ELEMENT SOLUTION}

T $\mathrm{n}$ order to obtain numerical results, we propose a higher-order plate element as shown in Fig. 2. The element has 9 nodes and each node has 5 degrees of freedom including the axial displacement $u_{0}$, lateral displacements $v_{0}, w_{0}$, and independent rotations $\psi_{x}, \psi_{y}$.

To obtain the generalized displacement corresponding to each degree of freedom inside an element, the Lagrange interpolation is used. This can be stated as:

$$
\begin{aligned}
& u_{0}(x, y)=\left[N_{u}\right]\{d\} \\
& v_{0}(x, y)=\left[N_{v}\right]\{d\} \\
& w_{0}(x, y)=\left[N_{w}\right]\{d\} \\
& \psi_{x}(x, y)=\left[N_{\psi_{x}}\right]\{d\} \\
& \psi_{y}(x, y)=\left[N_{\psi_{y}}\right]\{d\}
\end{aligned}
$$

where $\{d\}$ is the element nodal displacement vector and $\left[N_{u}\right],\left[N_{v}\right],\left[N_{w}\right],\left[N_{\psi_{x}}\right]$, and $\left[N_{\psi_{y}}\right]$ are the shape function matrices, defined as:

$$
\begin{aligned}
& {\left[N_{u}\right]=\left[\begin{array}{llllllllllllllll}
N_{1} & 0 & 0 & 0 & 0 & N_{2} & 0 & 0 & 0 & 0 & \ldots & N_{9} & 0 & 0 & 0 & 0
\end{array}\right]} \\
& {\left[N_{\nu}\right]=\left[\begin{array}{llllllllllllllll}
0 & N_{1} & 0 & 0 & 0 & 0 & N_{2} & 0 & 0 & 0 & \ldots & 0 & N_{9} & 0 & 0 & 0
\end{array}\right]} \\
& {\left[N_{w}\right]=\left[\begin{array}{llllllllllllllll}
0 & 0 & N_{1} & 0 & 0 & 0 & 0 & N_{2} & 0 & 0 & \ldots & 0 & 0 & N_{9} & 0 & 0
\end{array}\right]} \\
& {\left[\begin{array}{c}
N_{\psi_{x}}
\end{array}\right]=\left[\begin{array}{llllllllllllllll}
0 & 0 & 0 & N_{1} & 0 & 0 & 0 & 0 & N_{2} & 0 & \ldots & 0 & 0 & 0 & N_{9} & 0
\end{array}\right]} \\
& {\left[\begin{array}{c}
N_{\psi_{y}}
\end{array}\right]=\left[\begin{array}{llllllllllllllll}
0 & 0 & 0 & 0 & N_{1} & 0 & 0 & 0 & N_{2} & 0 & \ldots & 0 & 0 & 0 & 0 & N_{9}
\end{array}\right]}
\end{aligned}
$$

where the $N_{i}$ functions are

$$
\begin{aligned}
& N_{1}=(\zeta-1)(2 \zeta-1)(\eta-1)(2 \eta-1) \\
& N_{2}=-4 \zeta(\zeta-1)(\eta-1)(2 \eta-1) \\
& N_{3}=\zeta(2 \zeta-1)(\eta-1)(2 \eta-1) \\
& N_{4}=-4(\zeta-1)(2 \zeta-1) \eta(\eta-1) \\
& N_{5}=16 \zeta(\zeta-1) \eta(\eta-1)
\end{aligned}
$$




$$
\begin{aligned}
& N_{6}=-4 \zeta(2 \zeta-1) \eta(\eta-1) \\
& N_{7}=(\zeta-1)(2 \zeta-1) \eta(2 \eta-1) \\
& N_{8}=-4 \zeta(\zeta-1) \eta(2 \eta-1) \\
& N_{9}=\zeta(2 \zeta-1) \eta(2 \eta-1)
\end{aligned}
$$

Here, $\zeta=\frac{x}{a_{e}}$. and $\eta=\frac{y}{b_{e}}$ are non-dimensional element coordinates and $a_{e}$ and $b_{e}$ are the element length and width, respectively.

Substituting Eqn.12 into Eqn.5 and Eqn.6 respectively provides:

$$
\begin{aligned}
& U_{P}=\frac{1}{2}\{d\}^{T}\left[K_{e}\right]\{d\} \\
& T_{P}=\frac{1}{2}\{\dot{d}\}^{T}\left[M_{e}\right]\{\dot{d}\}
\end{aligned}
$$

where $\left[K_{e}\right]$ is the element stiffness matrix and $\left[M_{e}\right]$ is the element mass matrix. Their expressions are provided in Appendix A. After assembling the element matrices and applying the Euler-Lagrange equations, the coupled governing equations of motion are obtained as follows:

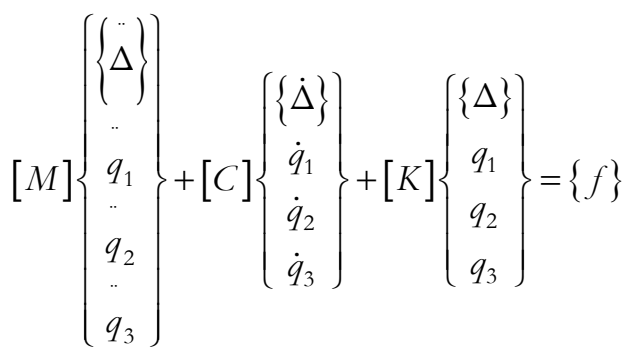

where $\{\Delta\}$ is the plate total displacement vector and $\left[C_{P}\right]$ is the plate damping matrix, assumed as Rayleigh's proportional damping [25]:

$$
\begin{aligned}
& {\left[C_{P}\right]=a_{0}\left[M_{P}\right]+a_{1}\left[K_{P}\right]} \\
& \frac{1}{2}\left[\begin{array}{cc}
1 / \omega_{i} & \omega_{i} \\
1 / \omega_{j} & \omega_{j}
\end{array}\right]\left\{\begin{array}{l}
a_{0} \\
a_{1}
\end{array}\right\}=\left\{\begin{array}{l}
\xi_{i} \\
\xi_{j}
\end{array}\right\}
\end{aligned}
$$

Eqn.17 is discretized by applying the Newmark time integration method in which $\gamma=1 / 2$ and $\beta=1 / 4$.

\section{NUMERICAL RESULTS}

$\mathrm{I}$

$\mathrm{n}$ this section, firstly, the free vibration results are compared with those available in the literature. Also, a parametric analysis is carried out to study the effects of system dynamic characteristics on the dynamic response of plate. The examples provided are based on the following material properties unless mentioned otherwise: 


$$
\begin{aligned}
& E_{1}=40 G P a, E_{2}=9.65 G P a, G_{12}=G_{13}=0.6 E_{2} \\
& G_{23}=0.5 E_{2}, \quad v_{12}=0.25, \quad \rho=1389.23\left(\mathrm{~kg} / \mathrm{m}^{3}\right)
\end{aligned}
$$

As a first example, the variation of the non-dimensional natural frequency of a laminated composite plate simply supported along all edges with symmetric cross-ply layup $\left(0^{\circ} / 90^{\circ}\right)$ is considered by changes of $a / b$. Tab. 1 shows the results.

\begin{tabular}{ccccc}
\hline & \multicolumn{5}{c}{$a / h$} \\
Refs. & 10 & 20 & 50 & 100 \\
Kant et al. [26] & 15.1048 & 17.6470 & 18.6720 & 18.835 \\
Matsunaga ]27] & 15.0721 & 17.6369 & 18.6702 & 18.835 \\
Reddy [28] & 15.1073 & 17.6457 & 18.6718 & 18.835 \\
Akavci [29] & 15.3684 & 17.7584 & 18.6934 & 18.841 \\
Rodriguez et al. [30] & 15.1674 & 17.7471 & 18.7895 & 18.956 \\
Abedi et al. [31] & 15.1056 & 17.6448 & 18.6719 & 18.836 \\
Present & 15.1425 & 17.6592 & 18.6689 & 18.789 \\
\hline
\end{tabular}

Table 1: Non-dimensional natural frequency $\left(\Omega=\left(\omega a^{2} / \mathrm{h}\right) \sqrt{\varrho / \mathrm{E}_{22}}\right)$ of a laminated composite plate simply supported at all edges with symmetric cross-ply layup $\left(0^{\circ} / 90^{\circ}\right)$.

From the above results, it is obvious that an increment in the plate length with respect to its thickness decreases the overall stiffness of the plate. As a consequence, it increases the non-dimensional natural frequency.

The next example expresses the effect of plate length to thickness on the first three non-dimensional natural frequencies. The plate has a $\left(0^{\circ} / 30^{\circ} / 60^{\circ} / 0^{\circ}\right)$ layup and two alternative boundary conditions: CCCC and SSSS.
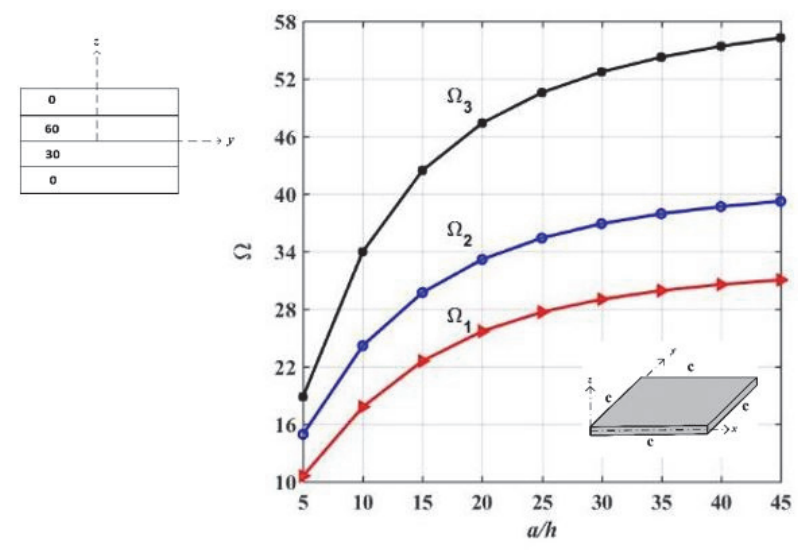

(a)

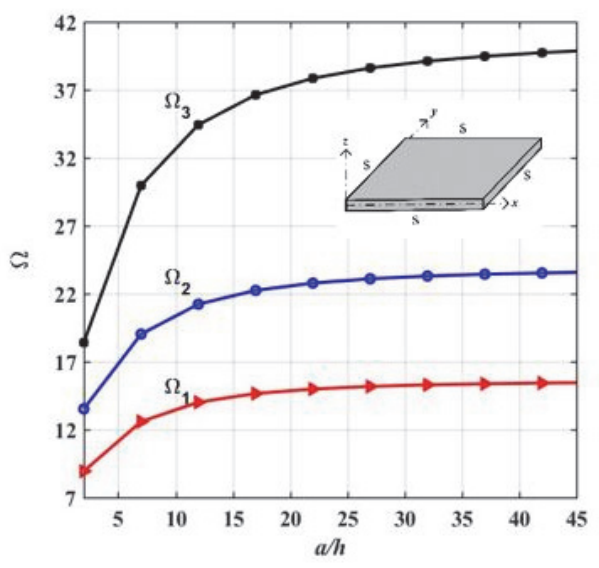

(b)

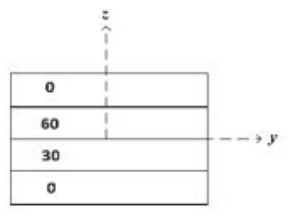

Figure 3: Effect of plate length to thickness on the first three non-dimensional natural frequencies (a) CCCC (b) SSSS. ( $E_{1}=25 E_{2}$, $\left.G_{12}=G_{13}=0.5 E_{2}, G_{23}=0.2 E_{2}\right)$ 
In order to obtain non-dimensional forced vibration results, firstly, non-dimensional parameters are discussed as below:

(a) velocity parameter $\alpha$, defined as the ratio of the fundamental period of the plate to the time required for the vehicle passing the span

$$
\alpha=\frac{2 \pi V}{\omega_{p} a}
$$

where $V$ is the velocity of vehicle. It is supposed that the velocity is constant and the vehicle is moving along the $x$-axis.

(b) mass parameter $\kappa$, defined as the ratio of vehicle mass to the plate mass

$$
\kappa=\frac{m}{M_{P}}=\frac{m}{\rho a b b}
$$

(c) frequency parameter $\gamma_{i}$, defined as the ratio of the natural frequency of quarter-vehicle to the fundamental frequency of the plate

$$
\gamma_{i}=\frac{\omega_{i}}{\omega_{P}}=\frac{\sqrt{\frac{k_{i}}{m / 4}}}{\omega_{P}} \quad(i=1,2,3,4)
$$

(d) mass moments of inertia $\varepsilon$ and $\chi$ are defined as below:

$$
\begin{aligned}
& \chi=\frac{I_{y}}{M_{P} a^{2}} \\
& \varepsilon=\frac{I_{x}}{M_{P} b^{2}}
\end{aligned}
$$

(e) logarithmic decrement of quarter-vehicle spring-dashpot $\Delta_{i}$, which is defined as

$$
\Delta_{i}=\frac{c_{i}}{2\left(\frac{m}{4}\right) f}=\frac{c_{i}}{2\left(\frac{m}{4}\right)\left(\frac{1}{2 \pi} \sqrt{\frac{k_{i}}{m / 4}}\right)}(i=1,2,3,4)
$$

In the following examples, the values of $k_{i}$ and $c_{i}$ for all suspension systems are equal.

To validate the forced vibration results, the model of the vehicle is reduced to a moving oscillator as discussed in [16]. Fig. 4 shows the comparison of results. It can be seen that the present reduced-order model tracks the reported results with low deviation. Therefore, the maximum errors are $2.29 \%$ for $\gamma=1.5$ and $2.17 \%$ for $\gamma=2$.

The next examples provide a better look at the mid-point deflection of the laminated composite plate. As a first example, the effect of boundary conditions on the dynamic magnification factor (DMF), defined as the ratio of maximum dynamic deflection with respect to maximum static deflection is studied. A laminated plate is considered with $\left(30^{\circ} / 60^{\circ}\right)_{A S}$ layup.

Here, the subscript " $A S$ " refers to an anti-symmetric stacking sequence. Four different boundary conditions are considered: CCCC, SSSS, CFCF, and SFSF. It should be noted that, for a better comparison among results, the maximum static deflection, in this example and in the further ones, is referred to the condition having lower DMF. It can be concluded that the CCCC boundary condition has higher stiffness. Thus, it gains lower amounts of DMF, as can be seen from Fig. 5. 


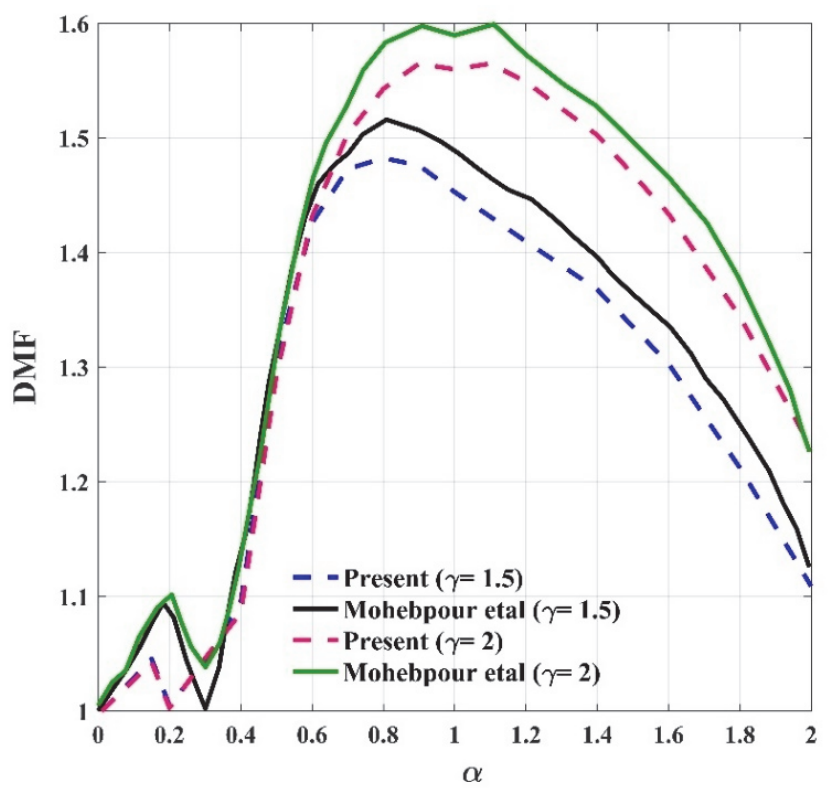

Figure 4: Comparison of present reduced-order model with reference [16]

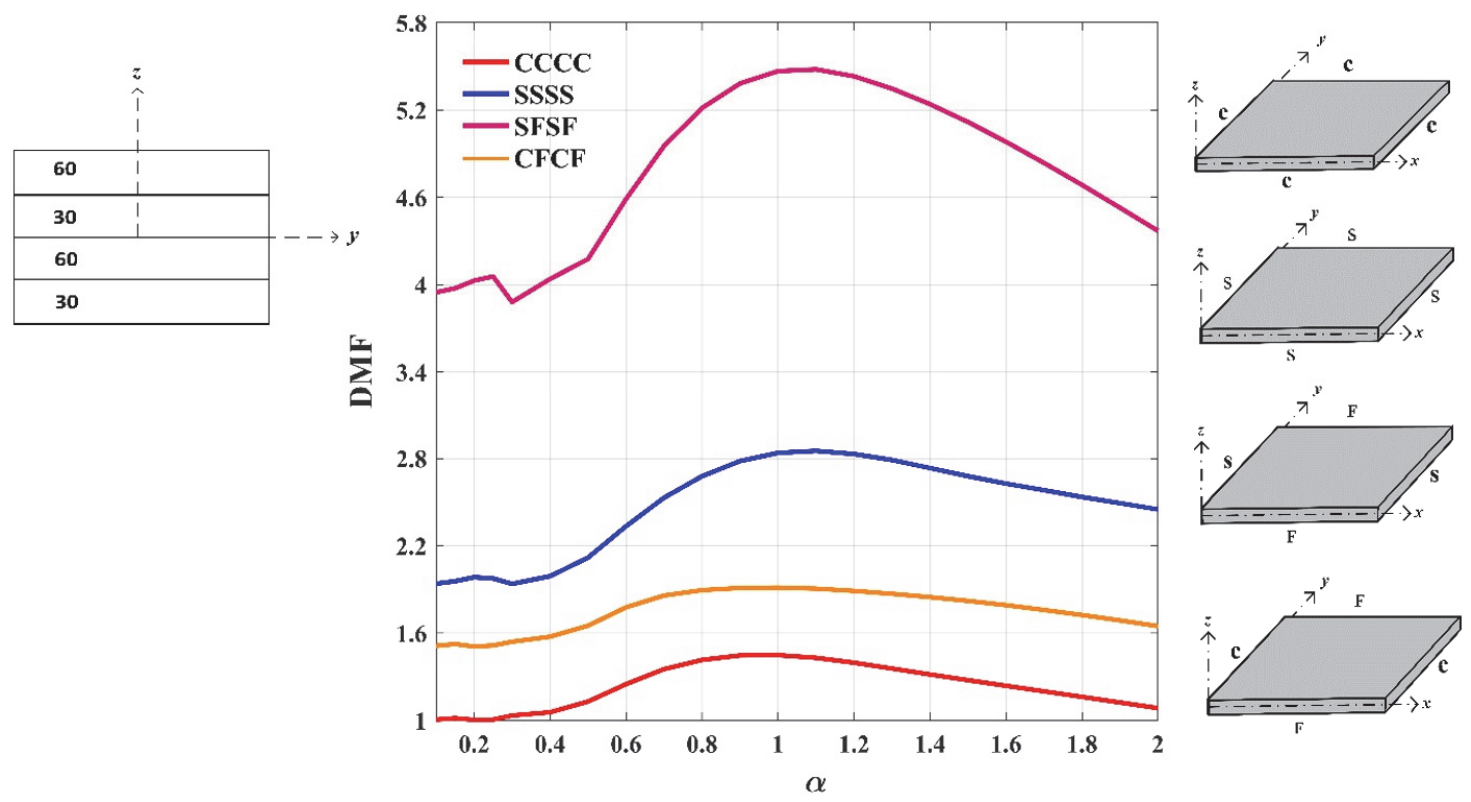

Figure 5: Influence of various boundary conditions on mid-point $\operatorname{DMF}\left(\kappa=0.2, \gamma=0.1, \chi=\varepsilon=0.1, \Delta=0.1, \xi_{P}=5 \%\right)$.

Fig. 6 presents the effect of the plate slenderness ratio parameter under CCCC boundary condition with $\left(30^{\circ} / 60^{\circ}\right)_{A S}$ layup. Increasing the length causes DMF to shift towards higher values. This happens because an increase in the length of the plate leads to a decrement in its structural stiffness and an increment in the dynamic deflection. To study the effect of plate damping ratio, consider a plate simply supported along all edges with $\left(30^{\circ} / 60^{\circ}\right)_{A S}$ layup. According to Fig. 7 increasing damping ratio leads to a reduction in mid-point dynamic deflection which indeed reduces DMF. However, the critical velocity is kept constant.

The last example expresses the influence of vehicle mass on the dynamic deflection of the mid-point. The laminated composite plate has $\left(0^{\circ} / 30^{\circ} / 60^{\circ} / 0^{\circ}\right)$ layup with CCCC boundary condition. Fig. 8 shows that, as long as the vehicle 
mass increases, the mid-point dynamic deflection increases during the existence of loading on plate. As a matter of fact, the DMF increases as shown in Fig. 9.

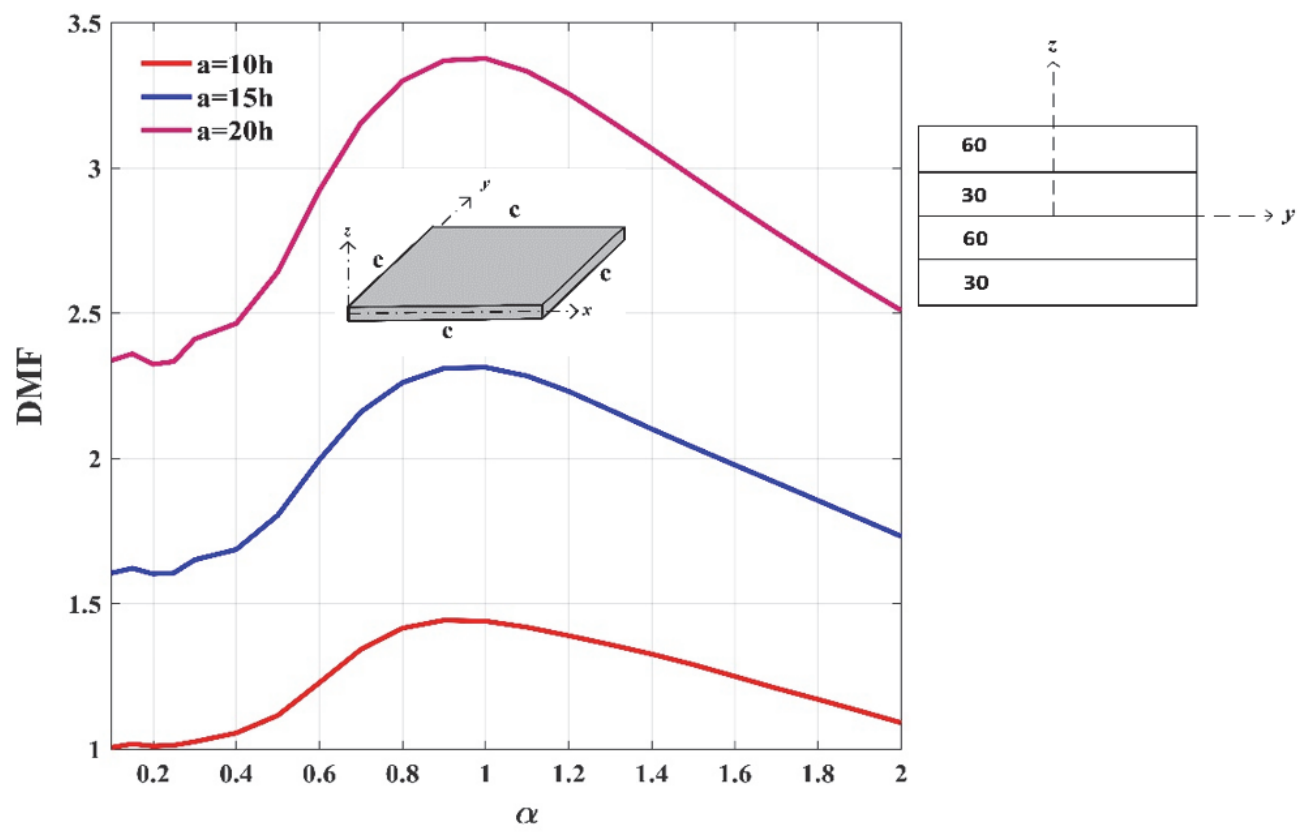

Figure 6: Influence of slenderness ratio on mid-point DMF ( $\kappa=0.2, \gamma=0.1, \chi=\varepsilon=0.1, \Delta=0.1, \quad \xi_{P}=5 \%$ ).

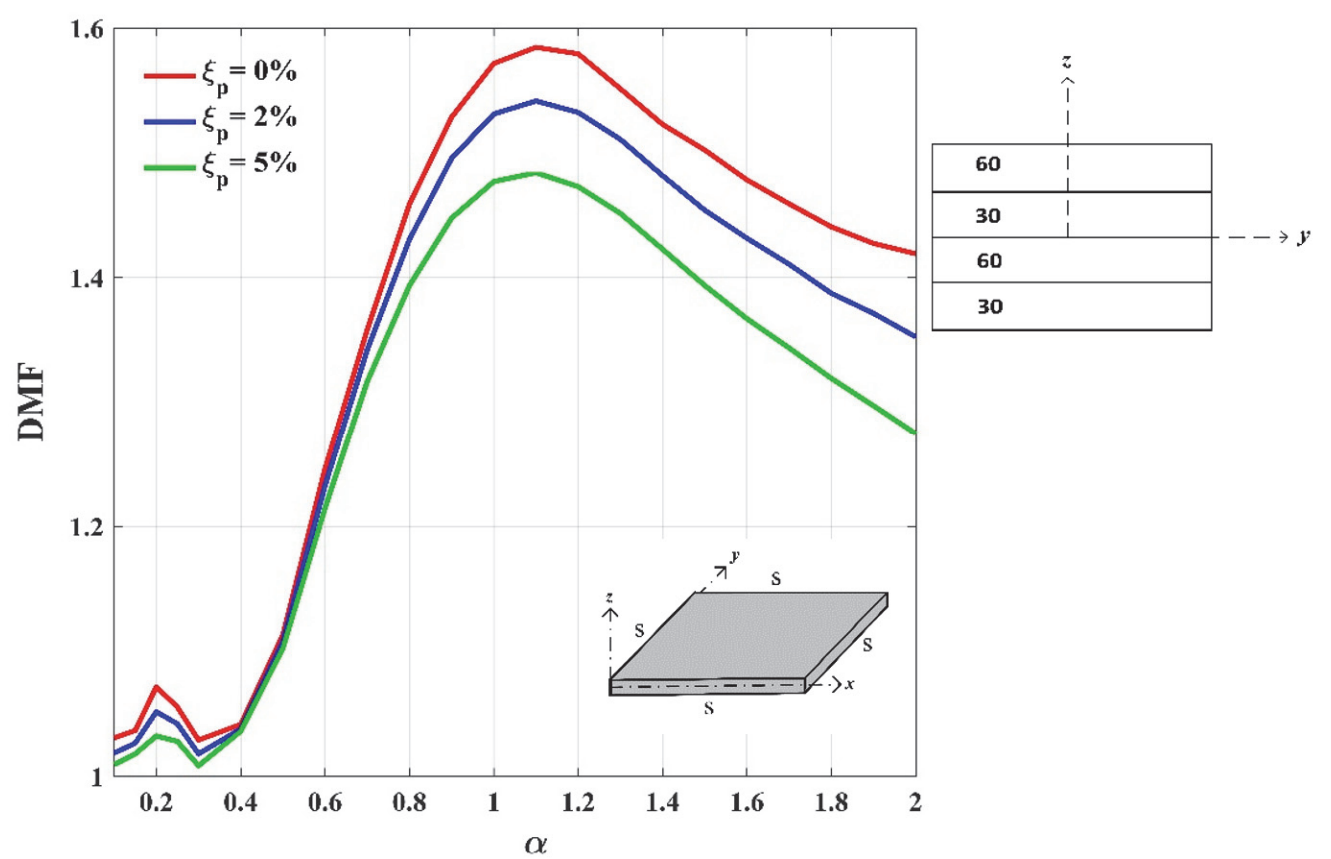

Figure 7: Effect of plate damping ratio on mid-point $\operatorname{DMF}(\kappa=0.2, \gamma=0.1, \chi=\varepsilon=0.1, \Delta=0.1)$.

\section{CONCLUSION}

T $\mathrm{n}$ this paper, the forced vibrations are investigated of a laminated composite plate under the action of a moving vehicle. Several examples are included to study the influence of system dynamic parameters, such as plate damping ratio, vehicle mass, and plate slenderness ratio. The equations of motion of plate are obtained based on first-order shear deformation 
theory and solved using Newmark's discretization scheme. Free and forced vibration results show good agreement with those available in the literature. This modeling algorithm can be extended to a full vehicle model to obtain results that are applicable in practical designs. It is shown that the SFSF boundary condition provides higher values of DMF. Besides, decreasing the damping ratio and increasing the slenderness ratio, alongside with the vehicle mass, can have considerable effect on the DMF.

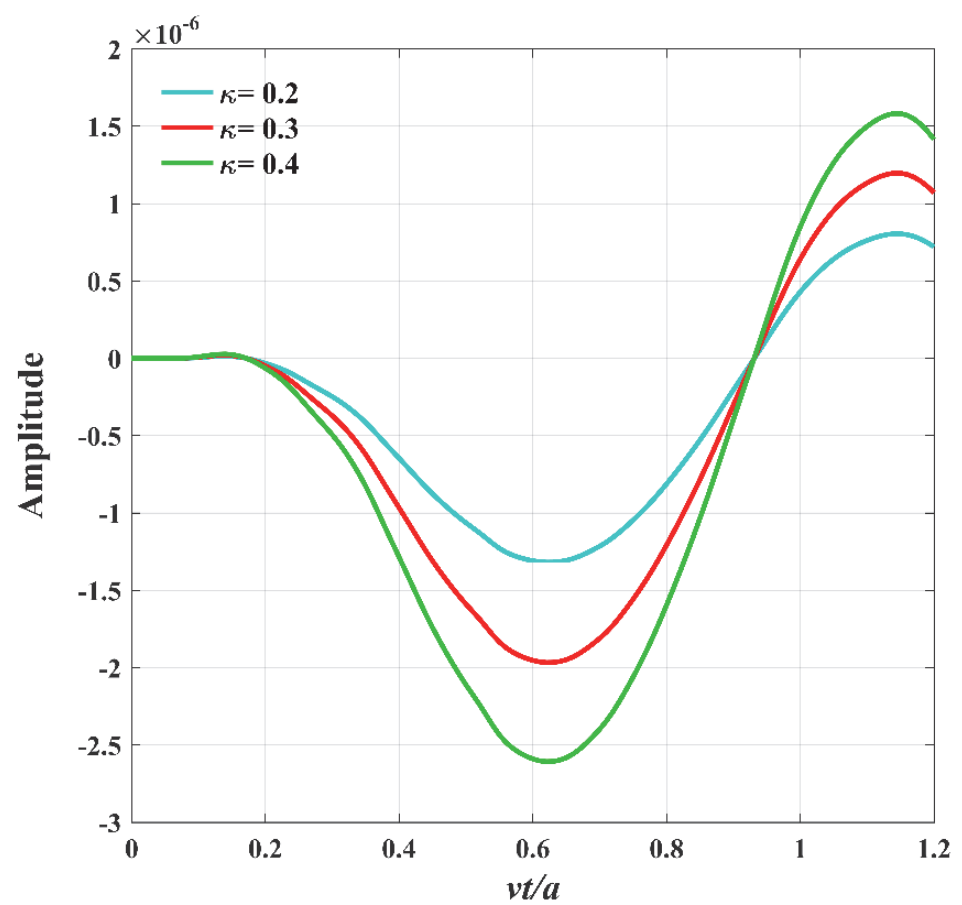

Figure 8: Effect of vehicle mass on mid-point dynamic deflection during the existence of load on plate $(\kappa=0.2 \gamma=0.1$, $\left.\chi=\varepsilon=0.1, \Delta=0.1, \xi_{p}=5 \%, \alpha=1\right)$

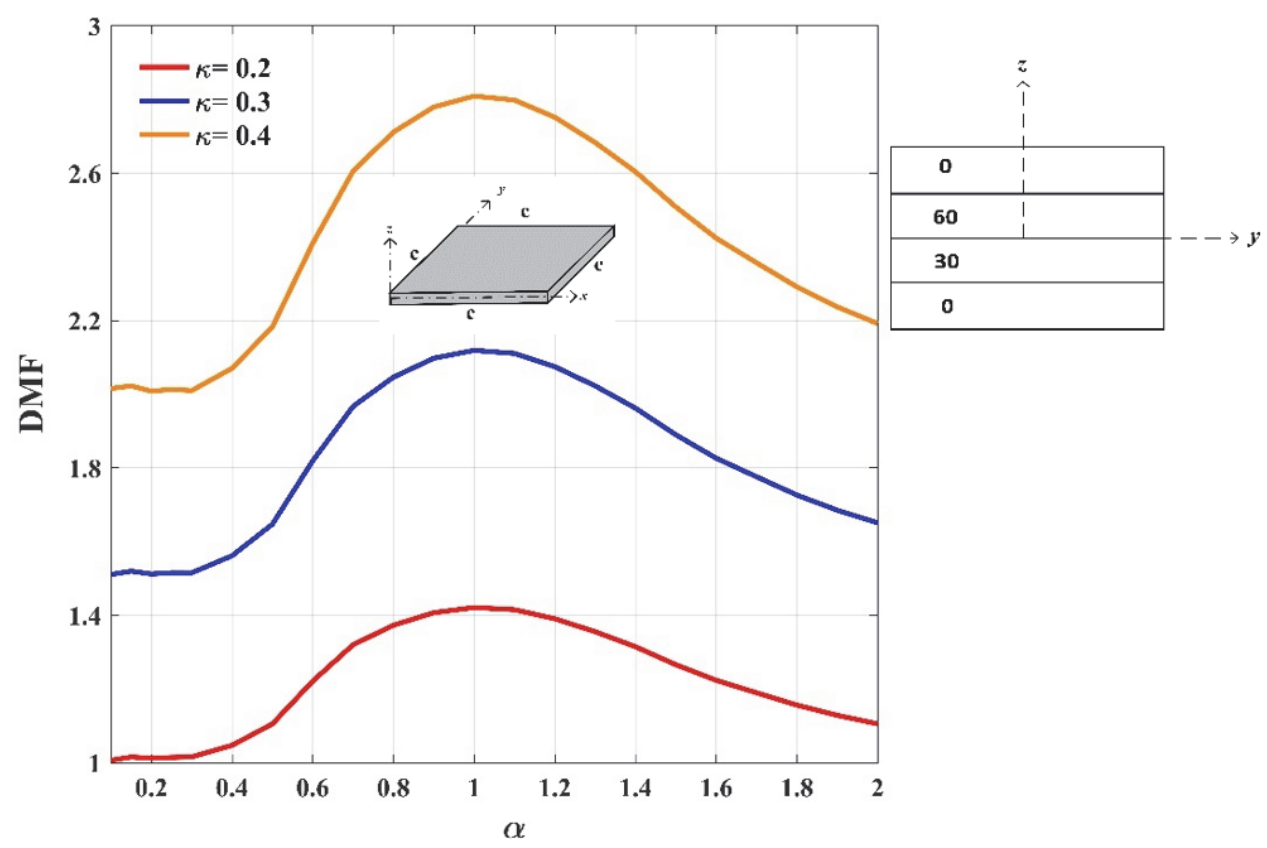

Figure 9: Dynamic magnification factor for various vehicle masses $\left(\kappa=0.2, \gamma=0.1, \chi=\varepsilon=0.1, \Delta=0.1, \quad \xi_{P}=5 \%\right)$ 


\section{APPENDix A}

$$
\begin{aligned}
& {\left[K_{\ell}\right]=\int_{0}^{a} \int_{0}^{b}\left[A_{11} N_{u_{0}, x}{ }^{T} \cdot N_{u_{0}, x}+A_{22} N_{v_{0}, y}{ }^{T} \cdot N_{v_{0}, y}+A_{66}\left(N_{u_{0}, y}{ }^{T} \cdot N_{u_{0}, y}+N_{v_{0}, x}{ }^{T} \cdot N_{v_{0}, x}\right.\right.} \\
& \left.+N_{u_{0}, y}{ }^{T} \cdot N_{v_{0}, x}+N_{v_{0}, x}{ }^{T} \cdot N_{u_{0}, y}\right)+D_{11} N_{\psi_{x}, x}{ }^{T} \cdot N_{\psi_{x}, x}+D_{22} N_{\psi_{y}, y}{ }^{T} \cdot N_{\psi_{y}, y} \\
& +D_{66}\left(N_{\psi_{x}, y}{ }^{T} \cdot N_{\psi_{x}, y}+N_{\psi_{y}, x}{ }^{T} \cdot N_{\psi_{y}, x}+N_{\psi_{x}, y}{ }^{T} \cdot N_{\psi_{y}, x}+N_{\psi_{x}, y}{ }^{T} \cdot N_{\psi_{y}, x}\right. \\
& \left.+N_{\psi_{y}, x}{ }^{T} \cdot N_{\psi_{x}, y}\right)+A_{44}\left(N_{\psi_{y}}{ }^{T} \cdot N_{\psi_{y}}+N_{w_{0}, y}{ }^{T} \cdot N_{w_{0}, y}+N_{\psi_{y}}{ }^{T} \cdot N_{w_{0}, y}\right. \\
& \left.+N_{w_{0}, y}{ }^{T} \cdot N_{\psi_{y}}\right)+A_{55}\left(N_{\psi_{x}}{ }^{T} \cdot N_{\psi_{x}}+N_{w_{0}, x}{ }^{T} \cdot N_{w_{0}, x}+N_{\psi_{x}}{ }^{T} \cdot N_{w_{0}, x}\right. \\
& \left.+N_{w_{0}, x}{ }^{T} \cdot N_{\psi_{x}}\right)+A_{12}\left(N_{u_{0}, x}{ }^{T} \cdot N_{v_{0}, y}+N_{v_{0}, y}{ }^{T} \cdot N_{u_{0}, x}\right)+A_{16}\left(N_{u_{0}, y}{ }^{T} \cdot N_{u_{0}, x}\right. \\
& \left.+N_{u_{0}, x}{ }^{T} \cdot N_{u_{0}, y}+N_{v_{0}, x}{ }^{T} \cdot N_{u_{0}, x}+N_{u_{0}, x}{ }^{T} \cdot N_{\nu_{0}, x}\right)+B_{11}\left(N_{\psi_{x}, x}{ }^{T} \cdot N_{u_{0}, x}+N_{u_{0}, x}{ }^{T} \cdot N_{\psi_{x}, x}\right) \\
& +B_{12}\left(N_{\psi_{y}, y}{ }^{T} \cdot N_{u_{0}, x}+N_{u_{0}, x}{ }^{T} \cdot N_{\psi_{y}, y}\right)+B_{16}\left(N_{\psi_{x}, y}{ }^{T} \cdot N_{u_{0}, x}+N_{u_{0}, x}{ }^{T} \cdot N_{\psi_{x}, y}\right. \\
& \left.+N_{\psi_{y}, x}{ }^{T} \cdot N_{u_{0}, x}+N_{u_{0}, x}{ }^{T} \cdot N_{\psi_{y}, x}\right)+A_{26}\left(N_{u_{0}, y}{ }^{T} \cdot N_{v_{0}, y}+N_{v_{0}, y}{ }^{T} \cdot N_{u_{0}, y}\right. \\
& \left.+N_{v_{0}, x}{ }^{T} \cdot N_{v_{0}, y}+N_{v_{0}, y}{ }^{T} \cdot N_{\nu_{0}, x}\right)+B_{12}\left(N_{\psi_{x}, x}{ }^{T} \cdot N_{\nu_{0}, y}+N_{\nu_{0}, y}{ }^{T} \cdot N_{\psi_{x}, x}\right) \\
& +B_{22}\left(N_{\psi_{y}, y}{ }^{T} \cdot N_{v_{0}, y}+N_{\nu_{0}, y}{ }^{T} \cdot N_{\psi_{y}, y}\right)+B_{26}\left(N_{\psi_{x}, y}{ }^{T} \cdot N_{\nu_{0}, y}+N_{v_{0}, y}{ }^{T} \cdot N_{\psi_{x}, y}\right. \\
& \left.+N_{\psi_{y}, x}{ }^{T} \cdot N_{v_{0}, y}+N_{v_{0}, y}{ }^{T} \cdot N_{\psi_{y}, x}\right)+B_{16}\left(N_{\psi_{x}, x}{ }^{T} \cdot N_{u_{0}, y}+N_{u_{0}, y}{ }^{T} \cdot N_{\psi_{x}, x}\right. \\
& \left.+N_{\psi_{x}, x}{ }^{T} \cdot N_{\nu_{0}, x}+N_{\nu_{0}, x}{ }^{T} \cdot N_{\psi_{x}, x}\right)+B_{26}\left(N_{\psi_{y}, y}{ }^{T} \cdot N_{u_{0}, y}+N_{u_{0}, y}{ }^{T} \cdot N_{\psi_{y}, y}\right. \\
& +N_{\psi_{y}, y}{ }^{T} \cdot N_{v_{0}, x}+B_{66}\left(N_{\psi_{x}, y}{ }^{T} \cdot N_{u_{0}, y}+N_{u_{0}, y}{ }^{T} \cdot N_{\psi_{x}, y}+N_{\psi_{x}, y}{ }^{T} \cdot N_{v_{0}, x}\right. \\
& +N_{v_{0}, x}{ }^{T} \cdot N_{\psi_{x}, y}+N_{\psi_{y}, x}{ }^{T} \cdot N_{u_{0}, y}+N_{u_{0}, y}{ }^{T} \cdot N_{\psi_{y}, x}+N_{\psi_{y}, x}{ }^{T} \cdot N_{v_{0}, x} \\
& \left.+N_{v_{0}, x}{ }^{T} \cdot N_{\psi_{y}, x}\right)+D_{12}\left(N_{\psi_{y}, y}{ }^{T} \cdot N_{\psi_{x}, x}+N_{\psi_{x}, x}{ }^{T} \cdot N_{\psi_{y}, y}\right)+D_{16}\left(N_{\psi_{x}, y}{ }^{T} \cdot N_{\psi_{x}, x}\right. \\
& \left.+N_{\psi_{x}, x}{ }^{T} \cdot N_{\psi_{x}, y}+N_{\psi_{y}, x}{ }^{T} \cdot N_{\psi_{x}, x}+N_{\psi_{x}, x}{ }^{T} \cdot N_{\psi_{y}, x}\right)+D_{26}\left(N_{\psi_{x}, y}{ }^{T} \cdot N_{\psi_{y}, y}\right. \\
& \left.+N_{\psi_{y}, y}{ }^{T} \cdot N_{\psi_{x}, y}+N_{\psi_{y}, x}{ }^{T} \cdot N_{\psi_{y}, y}+N_{\psi_{y}, y}{ }^{T} \cdot N_{\psi_{y}, x}\right)+A_{45}\left(N_{\psi_{x}}{ }^{T} \cdot N_{\psi_{y}}\right. \\
& +N_{\psi_{y}}{ }^{T} \cdot N_{\psi_{x}}+N_{\psi_{x}}{ }^{T} \cdot N_{w_{0}, y}+N_{w_{0}, y}{ }^{T} \cdot N_{\psi_{x}}+N_{w_{0}, x}{ }^{T} \cdot N_{\psi_{y}}+N_{\psi_{y}}{ }^{T} \cdot N_{w_{0}, x} \\
& \left.\left.+N_{w_{0}, x}{ }^{T} \cdot N_{w_{0}, y}+N_{w_{0}, y}{ }^{T} \cdot N_{w_{0}, x}\right)\right] d y d x
\end{aligned}
$$$$
\begin{aligned}
{\left[M_{e}\right] } & =\int_{0}^{a} \int_{0}^{b}\left[\mathrm{I}_{0}\left(\left[N_{u}\right]^{T} \cdot\left[N_{u}\right]+\left[N_{v}\right]^{T} \cdot\left[N_{\nu}\right]+\left[N_{w}\right]^{T} \cdot\left[N_{w}\right]\right)+\mathrm{I}_{1}\left(\left[N_{u}\right]^{T} \cdot\left[N_{\psi_{x}}\right]+\left[N_{\psi_{x}}\right]^{T} \cdot\left[N_{u}\right]\right.\right. \\
& \left.\left.\left.+\left[N_{v}\right]^{T} \cdot\left[N_{\psi_{y}}\right]+\left[N_{\psi_{y}}\right]^{T} \cdot\left[N_{v}\right]\right)\right)+\mathrm{I}_{2}\left(\left[N_{\psi_{x}}\right]^{T} \cdot\left[N_{\psi_{x}}\right]+\left[N_{\psi_{y}}\right]^{T} \cdot\left[N_{\psi_{y}}\right]\right)\right] d y d x
\end{aligned}
$$

\section{REFERENCES}

[1] Song, Q., Shi, J., Liu, Z., Wan, Y. (2016). Dynamic analysis of rectangular thin plates of arbitrary boundary conditions under moving loads, Int. J. Mech. Sci., 117, pp. 16-29, DOI: 10.1016/j.ijmecsci.2016.08.005.

[2] Frýba, L. (2013). Vibration of solids and structures under moving loads, Vol. 1. Springer Science \& Business Media.

[3] Kim, T., Lee, U. (2018). Vibration analysis of thin plate structures subjected to a moving force using frequency-domain spectral element method, Shock Vib., DOI: 10.1155/2018/1908508. 
[4] Babagi, P.N., Navayi Neya, B., Dehestani, M. (2017). Three dimensional solution of thick rectangular simply supported plates under a moving load, Meccanica, 52(15), pp. 3675-3692, DOI: 10.1007/s11012-017-0653-x.

[5] Wu, J.J. (2007). Vibration analyses of an inclined flat plate subjected to moving loads, J. Sound Vib., 299(1-2), pp. 373387, DOI: 10.1016/j.jsv.2006.07.002.

[6] Mofid, M., Shadnam, M. (2000). On the response of beams with internal hinges, under moving mass, Adv. Eng. Softw., 31(5), pp. 323-328, DOI: 10.1016/S0965-9978(99)00061-7.

[7] Esen, I. (2013). A new finite element for transverse vibration of rectangular thin plates under a moving mass, Finite Elem. Anal. Des., 66, pp. 26-35, DOI: 10.1016/j.finel.2012.11.005.

[8] Esen, I. (2011). Dynamic response of a beam due to an accelerating moving mass using moving finite element approximation, Math. Comput. Appl., 16(1), pp. 171-82, DOI: 10.3390/mca16010171.

[9] Moghaddas, M., Sedaghati, R., Esmailzadeh, E., Khosravi, P. (2009). Finite element analysis of a Timoshenko beam traversed by a moving vehicle, Proc. Inst. Mech. Eng. Part K J. Multi-Body Dyn., 223(3), pp. 231-243, DOI: $10.1243 / 14644193$ JMBD179.

[10] Ebrahimi, M., Gholampour, S., Jafarian Kafshgarkolaei, H., Nikbin, I.M. (2015). Dynamic behavior of a multispan continuous beam traversed by a moving oscillator, Acta Mech., 226(12), pp. 4247-4257, DOI: $10.1007 /$ s00707-015-1474-4.

[11] Pesterev, A.V., Yang, B., Bergman, L.A., Tan, C.A. (2001). Response of elastic continuum carrying multiple moving oscillators, J. Eng. Mech, 127.3, pp. 260-265.

[12] Ghafoori, E., Kargarnovin, M.H., Ghahremani, A.R. (2011). Dynamic responses of a rectangular plate under motion of an oscillator using a semi-analytical method, JVC/Journal Vib. Control, 17(9), pp. 1310-1324, DOI: $10.1177 / 1077546309358957$.

[13] Wu, J.J. (2006). Free vibration characteristics of a rectangular plate carrying multiple three-degree-of-freedom springmass systems using equivalent mass method, Int. J. Solids Struct., 43(3-4), pp. 727-746, DOI: $10.1016 /$ j.ijsolstr.2005.03.061.

[14] Lin, Y.H., Trethewey, M.W. (1990). Finite element analysis of elastic beams subjected to moving dynamic loads. JSV/Journal of sound and Vib., 136.2, pp. 323-342.

[15] Malekzadeh, P., Fiouz, A.R., Razi, H. (2009). Three-dimensional dynamic analysis of laminated composite plates subjected to moving load, Compos. Struct., 90(2), pp. 105-114, DOI: 10.1016/j.compstruct.2009.02.008.

[16] Mohebpour, S.R., Malekzadeh, P., Ahmadzadeh, A.A. (2011). Dynamic analysis of laminated composite plates subjected to a moving oscillator by FEM, Compos. Struct., 93(6), pp. 1574-1583, DOI: 10.1016/j.compstruct.2011.01.003.

[17] Lee, S.Y., Yhim, S.S. (2004). Dynamic analysis of composite plates subjected to multi-moving loads based on a third order theory, Int. J. Solids Struct., 41(16-17), pp. 4457-4472, DOI: 10.1016/j.ijsolstr.2004.03.021.

[18] Ghafoori, E., Asghari, M. (2010). Dynamic analysis of laminated composite plates traversed by a moving mass based on a first-order theory, Compos. Struct., 92(8), pp. 1865-1876, DOI: 10.1016/j.compstruct.2010.01.011.

[19] Mohebpour, S.R., Fiouz, A.R., Ahmadzadeh, A.A. (2011). Dynamic investigation of laminated composite beams with shear and rotary inertia effect subjected to the moving oscillators using FEM, Compos. Struct., 93(3), pp. 1118-1126, DOI: 10.1016/j.compstruct.2010.09.011.

[20] Kim, N. Il. (2010). Dynamic stability behavior of damped laminated beam subjected to uniformly distributed subtangential forces, Compos. Struct., 92(11), pp. 2768-2780, DOI: 10.1016/j.compstruct.2010.04.005.

[21] Saadatmorad, M., Jafari-Talookolaei, R.-A., Pashaei, M.H., Khatir, S. (2021). Damage detection on rectangular laminated composite plates using wavelet based convolutional neural network technique, Compos. Struct., 278, 114656, DOI: $10.1016 /$ j.compstruct.2021.114656.

[22] Khatir, S., Tiachacht, S., Thanh, C.L., Ghandourah, E., Mirjalili, S., Abdel Wahab, M. (2021). An improved Artificial Neural Network using Arithmetic Optimization Algorithm for damage assessment in FGM composite plates. Compos. Struct., 273, 114287, DOI: 10.1016/j.compstruct.2021.114287.

[23] Zenzen, R., Khatir, S., Belaidi, I., Thanh, C.L., Abdel Wahab, M. (2021). A modified transmissibility indicator and Artificial Neural Network for damage identification and quantification in laminated composite structures, Compos. Struct., 248, 112497, DOI: 10.1016/j.compstruct.2020.112497.

[24] Reddy, J.N. (2003). Mechanics of laminated composite plates and shells: theory and analysis, CRC press

[25] Chopra, A.K. (2012). Dynamics of structures, Upper Saddle River, NJ: Pearson Education

[26] Kant, T., Swaminathan, K. (2001). Analytical solutions for free vibration of laminated composite and sandwich plates based on a higher-order refined theory, Compos. Struct., 53(1), pp. 73-85, DOI: 10.1016/S0263-8223(00)00180-X.

[27] Matsunaga, H. (2000). Vibration and stability of cross-ply laminated composite plates according to a global higher-order plate theory, Compos. Struct., 48(4), pp. 231-244, DOI: 10.1016/S0263-8223(99)00110-5. 
[28] Reddy, J.N. (1984). A simple higher-order theory for laminated composite plates, J. Appl. Mech. Trans. ASME, 51(4), pp. 745-752, DOI: 10.1115/1.3167719.

[29] Akavci, S.S. (2007). Buckling and free vibration analysis of symmetric and antisymmetric laminated composite plates on an elastic foundation, J. Reinf. Plast. Compos., 26(18), pp. 1907-1919, DOI: 10.1177/0731684407081766.

[30] Rodrigues, J.D., Natarajan, S., Ferreira, A.J.M., Carrera, E., Cinefra, M., Bordas, S.P.A. (2014). Analysis of composite plates through cell-based smoothed finite element and 4-noded mixed interpolation of tensorial components techniques, Comput. Struct., 135, pp. 83-87, DOI: 10.1016/j.compstruc.2014.01.011.

[31] Abedi, M., Jafari-talookolaei, R., Valvo, P.S. (2016). A new solution method for free vibration analysis of rectangular laminated composite plates with general stacking sequences and edge restraints, Comput. Struct., 175, pp. 144-156, DOI: 10.1016/j.compstruc.2016.07.007. 